\title{
Evaluation of Salivary Glands Lesions Frequency in Pathology Centers of Hamadan Dental Faculty and Besat Hospital from 2006 to 2017
}

\section{Fahimeh Baghaei (PhD) ${ }^{1}$, Naser Mohammad Gholi Mezerji (MSc) ${ }^{2}$, Setareh Shojaei $(\mathrm{PhD})^{3, *}$, Milad Amirian (MD) ${ }^{4}$}

${ }^{1}$ Associate Professor, Department of Oral Pathology ,Dental Faculty, Hamadan University of Medical Sciences, Hamadan, Iran

${ }^{2}$ Ph.D Student in Biostatistics, Department of Biostatistics, Students Research Committee, Hamadan University of Medical Science, Hamadan, Iran

${ }^{3}$ Associate Professor, Department of Oral \& Maxillofacial Pathology, School of Dentistry, Hamadan University of Medical Sciences, Hamadan, Iran

${ }^{4}$ Dentist, Hamadan University of Medical Sciences, Hamadan, Iran

* Corresponding Author: Setareh Shojaei, Department of Oral \& Maxillofacial Pathology, School of Dentistry, Hamadan University of Medical Sciences, Hamadan, Iran. Email: s.shojaei.umsha@gmail.com

Received: 03/12/2018

Accepted: 13/02/2019

How to Cite this Article: Baghaei F, Mohammad Gholi Mezerji N, Shojaei S, Amirian M. Evaluation of Salivary Glands Lesions Frequency in Pathology centers of Hamadan Dental Faculty and Besat Hospital from 2006 to 2017. Pajouhan Scientific Journal. 2019; 17(2): 45-53. DOI: 10.29252/psj.17.2.45

\section{Abstract}

Background and Objective: Epidemiological study in Oral lesions and diseases can help to identify risk factors for preventing diseases. Therefore, the present study aimed to determine the frequency of salivary gland lesions in a pathology laboratory of Hamedan dental school and Besat Hospital.

Materials and Methods: In this descriptive cross-sectional study all records of patients from 2006 to 2017 were reviewed by referring to the Department of Pathology of Hamadan Dental School and Archives of Besat Hospital. The required data including histopathologic diagnosis and anatomic location of lesions, age and gender were recorded and the data were analyzed using SPSS v.16 statistical software.

Results: The results showed that salivary lesions comprise $6 / 63 \%$ of total head and neck lesions, of which $65.4 \%, 24.3 \%$ and $10.3 \%$ of lesions were non-neoplastic and benign and malignant neoplastic lesions respectively. Also, the forecasting of the incidence of cases for the next three years was at least $6.20 \%$ and a maximum of $10.36 \%$.

Conclusion: The results of this study suggested that future studies with more emphasis on clinicians will provide more accurate information about patients. Also, extensive studies are suggested in other cities and provinces with a longer period of time.

Keywords: Epidemiology; Hamadan; Salivary Gland Lesions 
dof: $10.29252 /$ psj.17.2.45

\section{فراوانى ضايعات غدد بزاقى در آزمايشكاه پاتولوزى دانشكده دندان بزشكى و بيمارستان

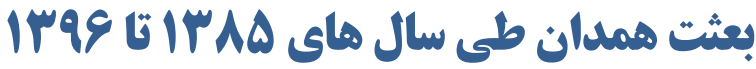

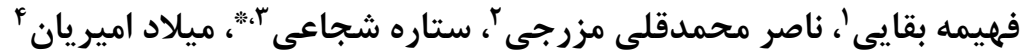

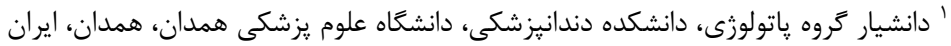

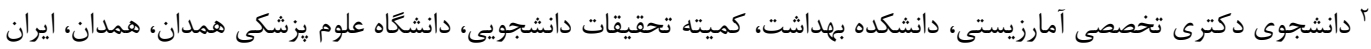

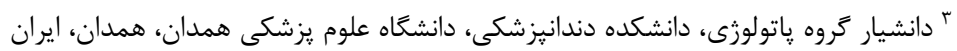

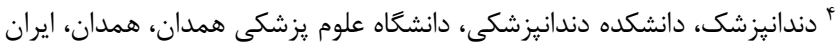
* *ويسنده مسئول: ستاره شجاعى، كروه ياتولوزى، دانشكده دندانيزشكى، دانشَاه علوم يزشكى همدان، همدان، ايران. ايميل: s.shojaei.umsha@gmail.com

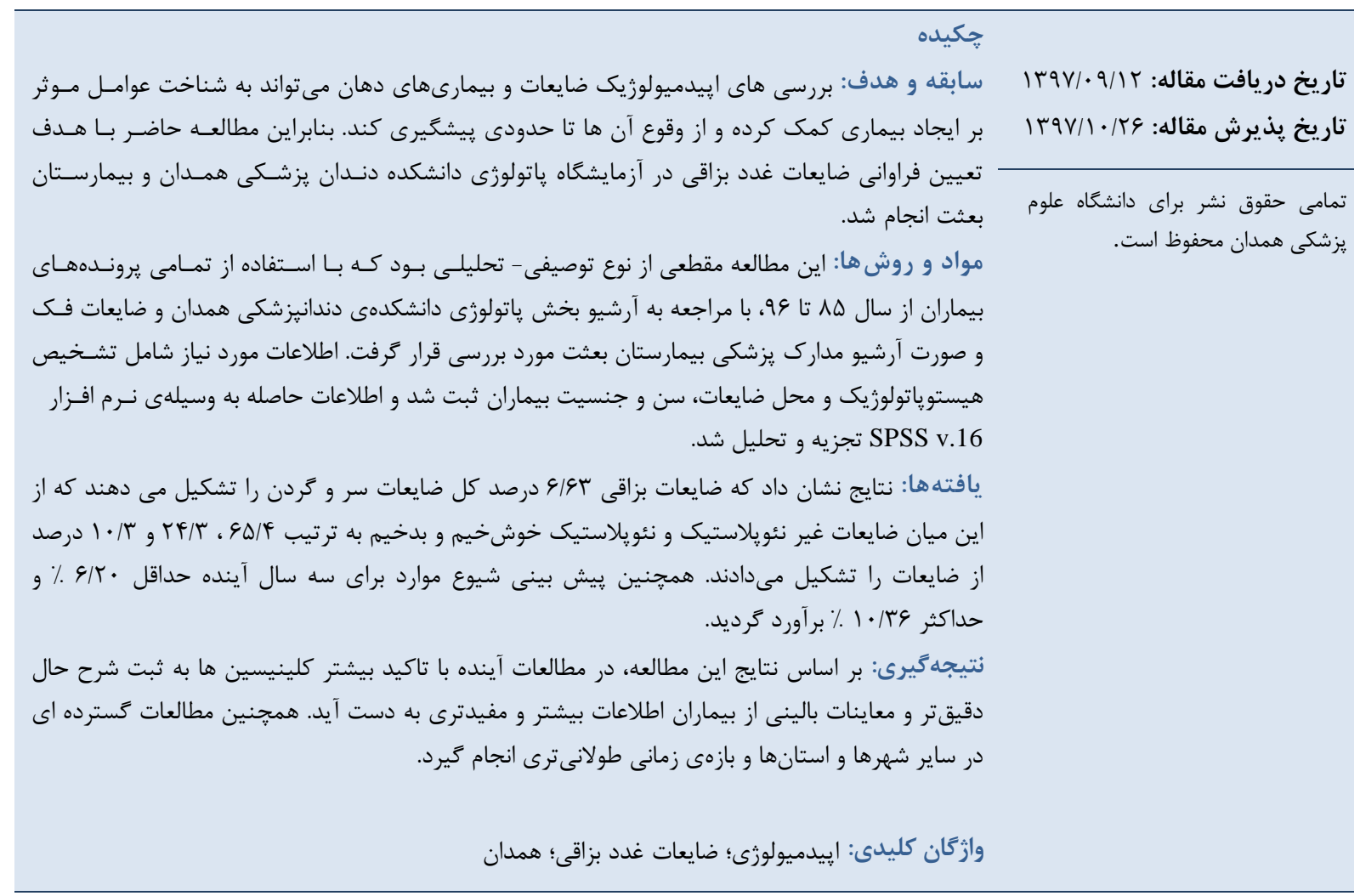

اتيولوزى اين ضايعات مىتواند تكاملى يا به علت التهاب، بيمارى

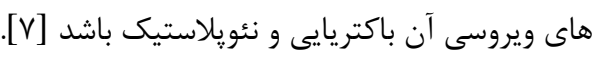

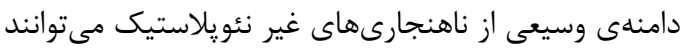

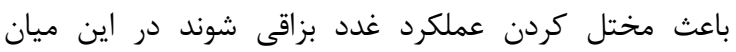

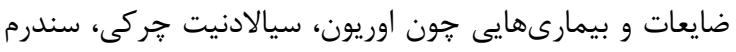

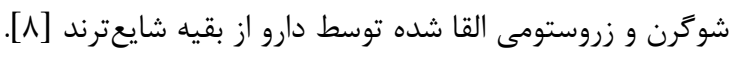

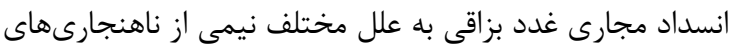

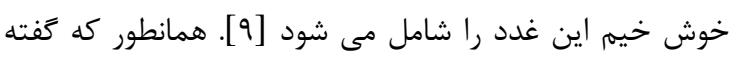

غدد بزاقى اصلى شامل غدد ياروتيد، تحت فكى و زير زبانى

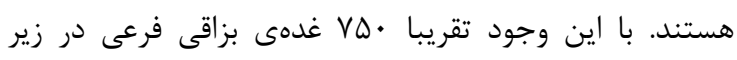

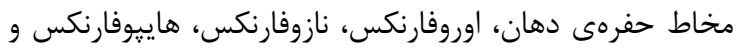

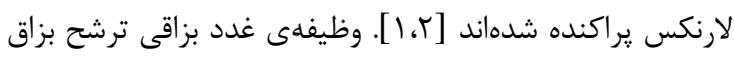
مى باشد كه نقش مهمى به عنوان لوبريكنت، كمكى به بلع بلع و و

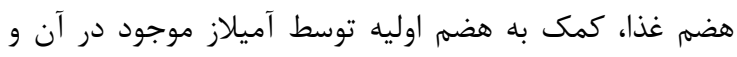

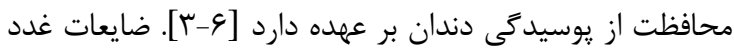

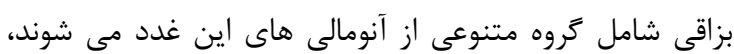


بررسى هاى إيدميولوزيك اين ضايعات مى تواند به

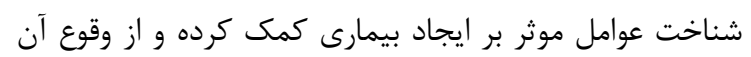

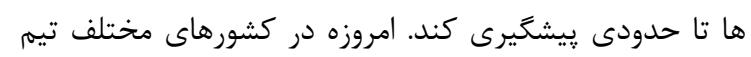

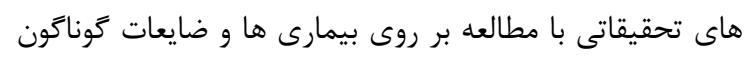

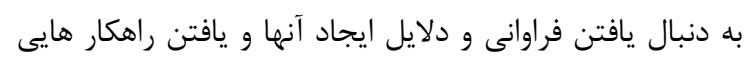

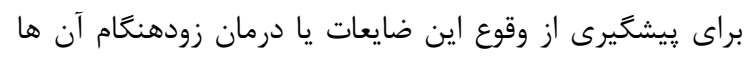

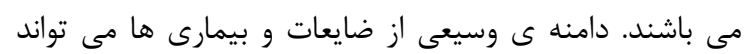

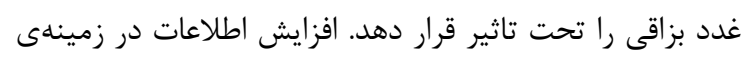

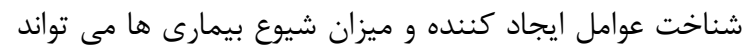

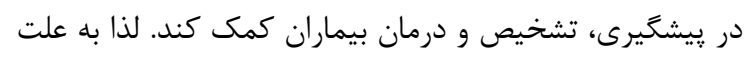

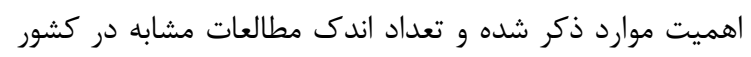

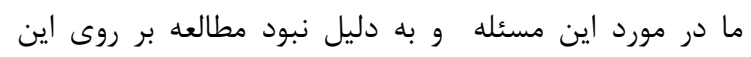
ضايعات در شهر همدان، مطالعه حاضر با هدف تعيين فراوانى دراني

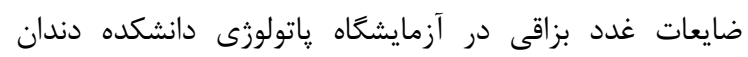

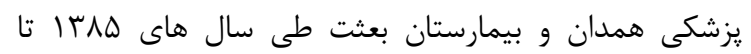

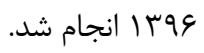

\section{مواد و روشها}

يزوهش حاضر يك مطالعه مقطعى از نوع توصيفى - تحليلى آلى

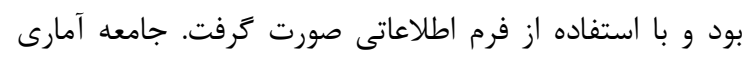

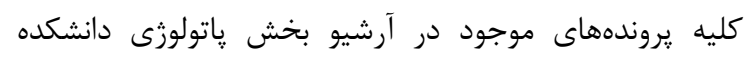

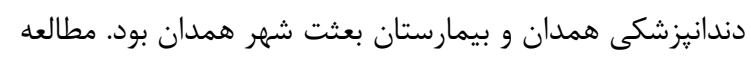

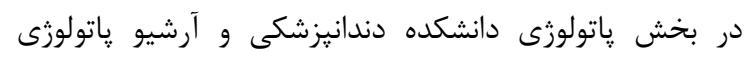

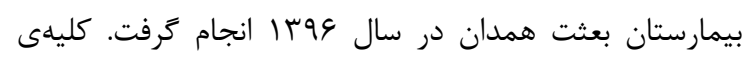

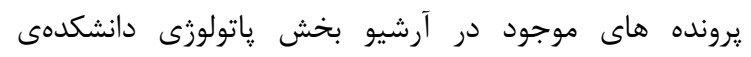

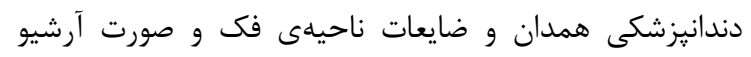

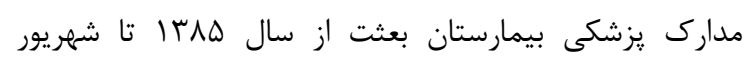

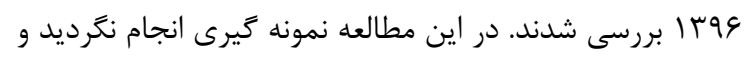

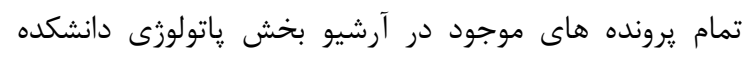

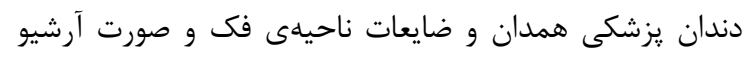

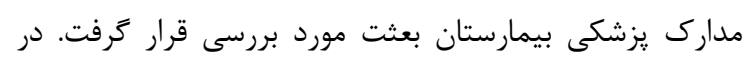

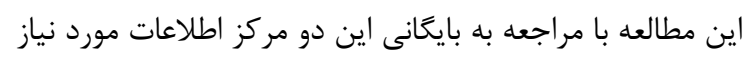

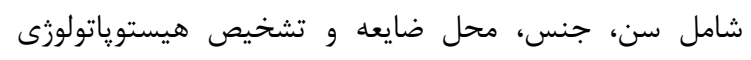

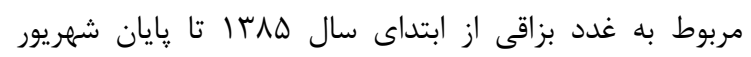

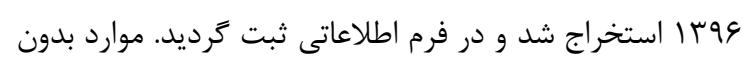

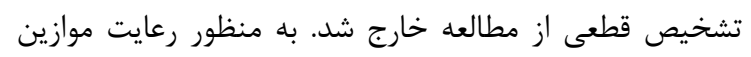

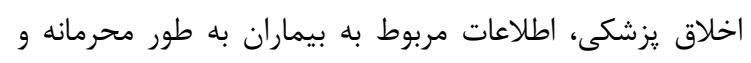

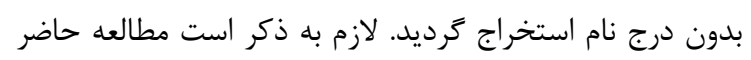

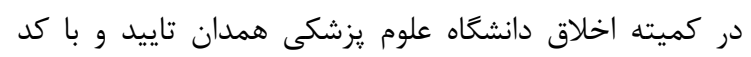

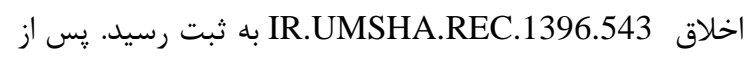
فهرست بندى و ثبت اطلاعات حاصله، آناليز آمارى با استفاده از

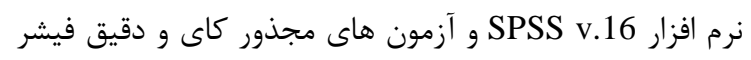
انجام كرفت. همجنين براى ييش بينى ها از روش

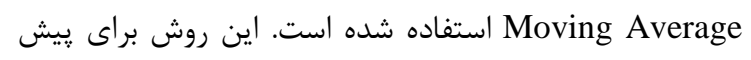

شد ضايعات غير نئويلاستيك به دلايل كَوناكَونى ايجاد مى إئى

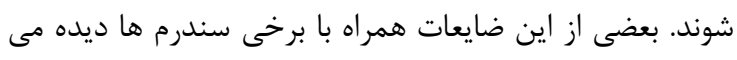

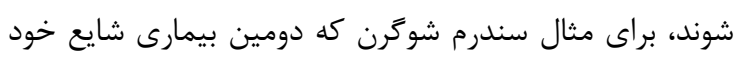

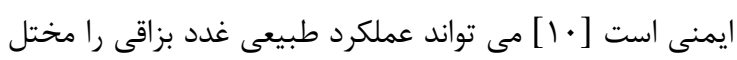

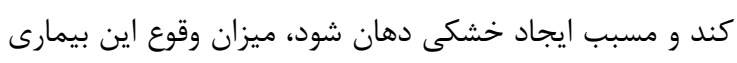

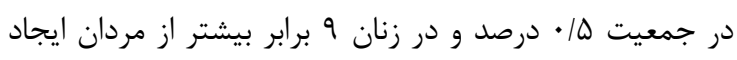
مى شود [11] [11]

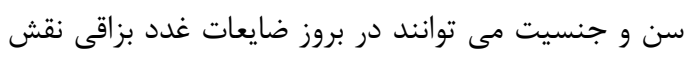

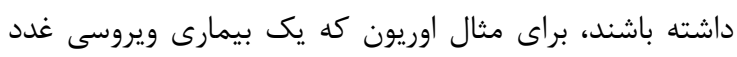

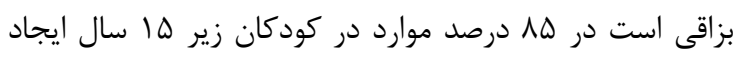

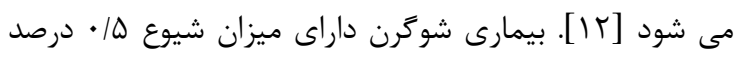

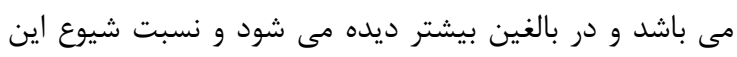

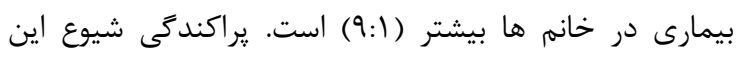

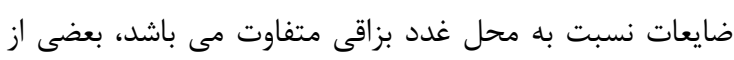

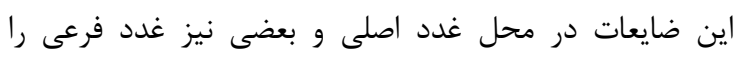

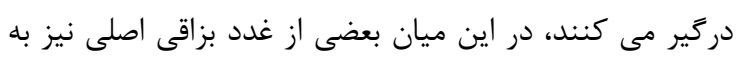

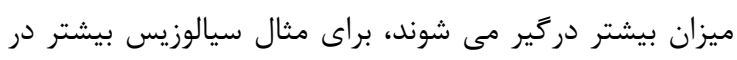

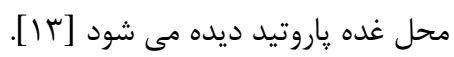

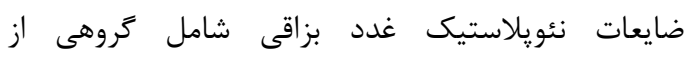

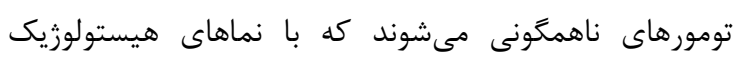

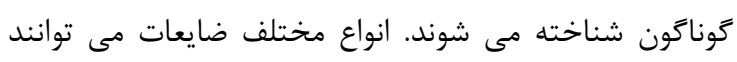

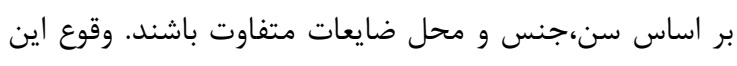

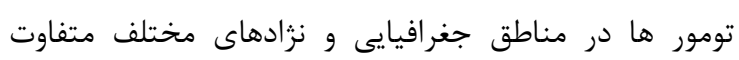

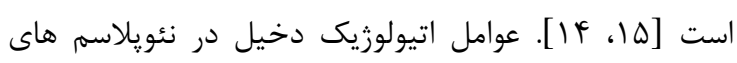

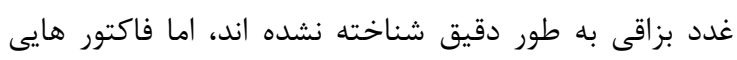

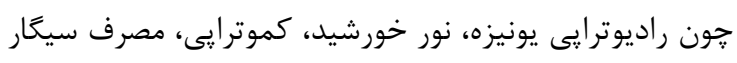

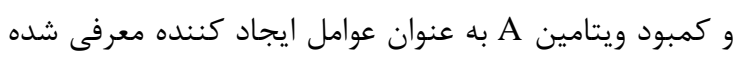

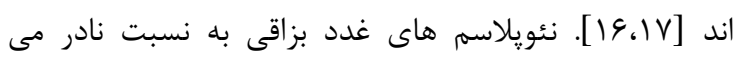

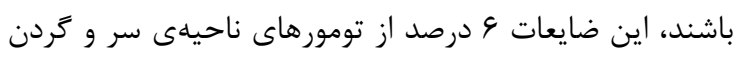

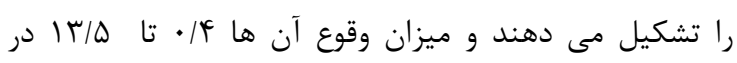

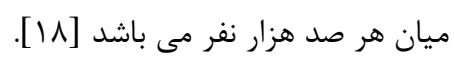

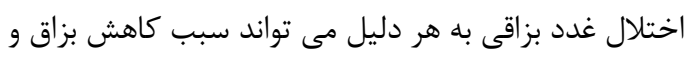

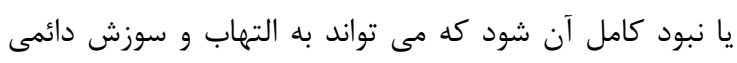

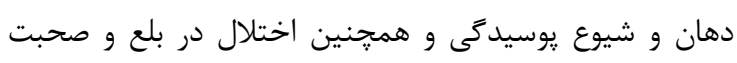

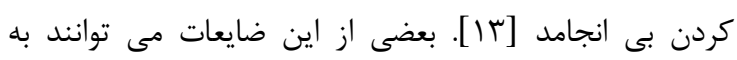

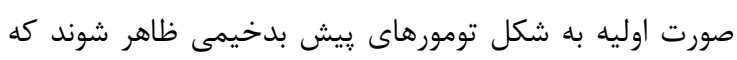

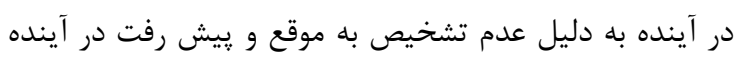

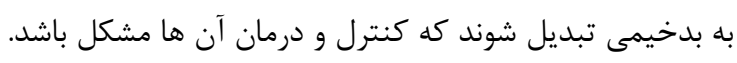

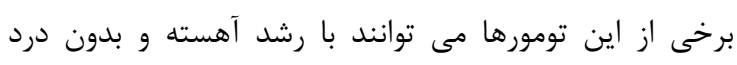

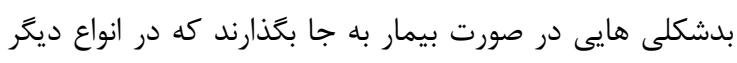

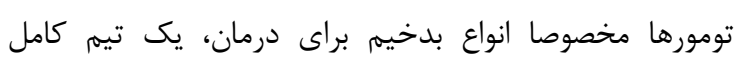

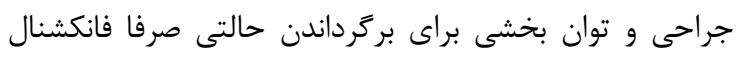

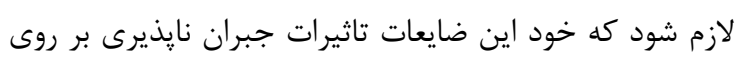

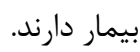


غيرنئويلاستيك بيشترين فراوانى را در دها هاى سوم و جهار

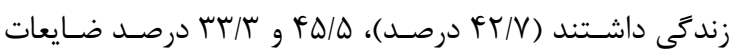

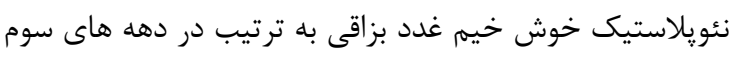

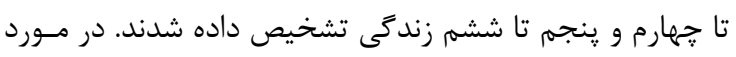

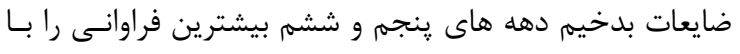

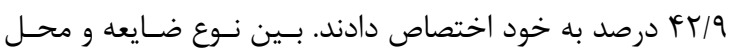

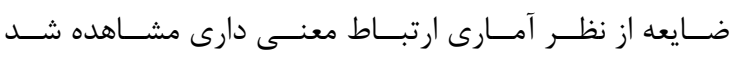

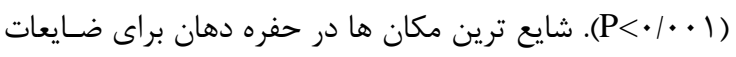

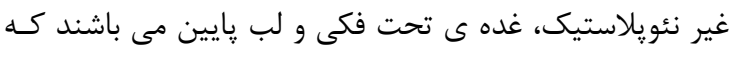

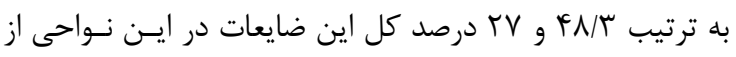

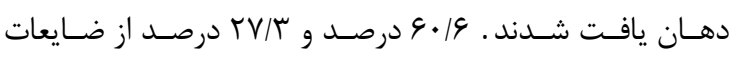

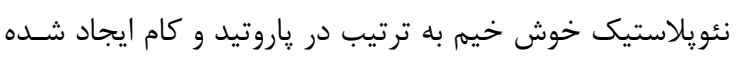

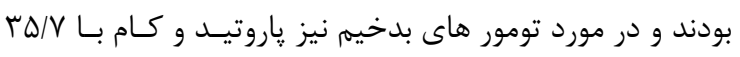

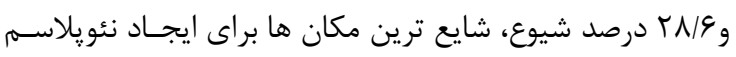

هاى بدخيم بودند ( جدول () ).

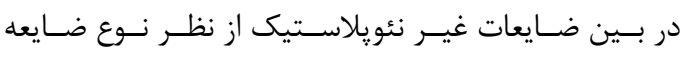

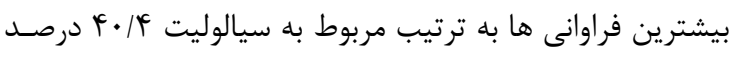

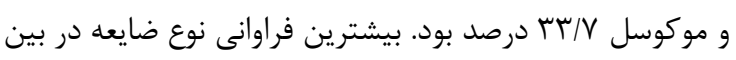

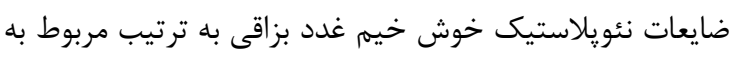

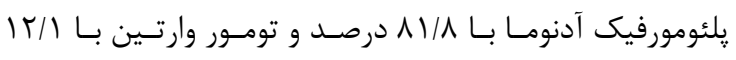

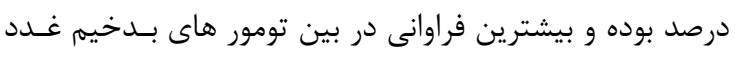

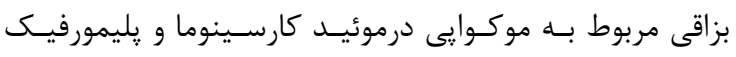

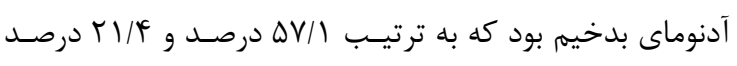

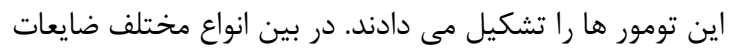

بينى داده هايى كه تنها شامل يك متغير تصادفى هستند، روند

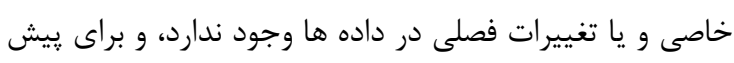
بين هاى كوتاه مدت مناسب است. سطح معنى دارى در در تمام آزمون ها هـ • إث در نظر كرفته شد. مدت مناست

يافتهها

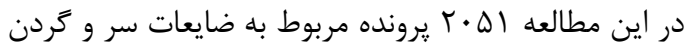

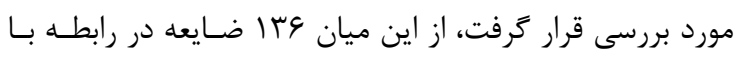

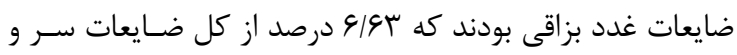

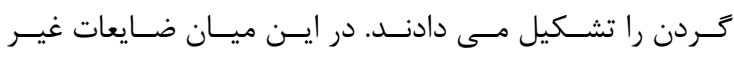
نئويلاستيك غدد بزاقى و

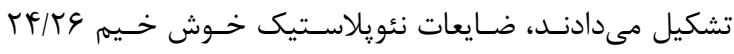

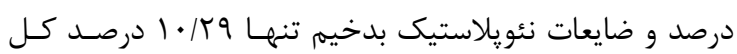

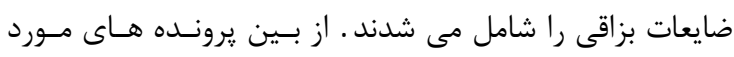

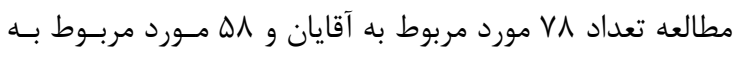

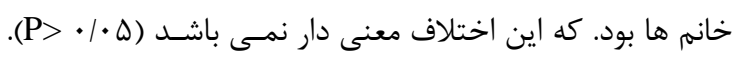

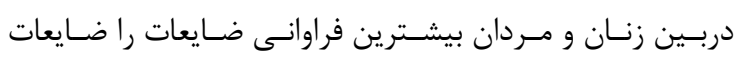

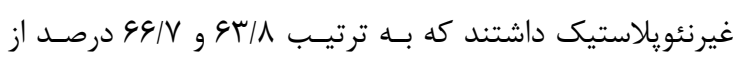

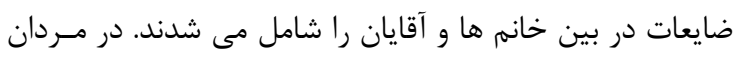

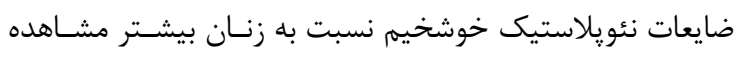

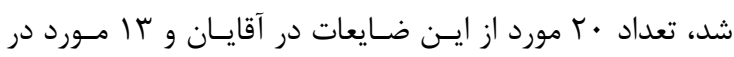

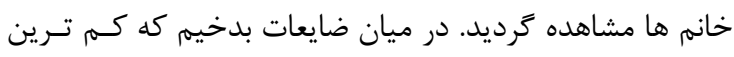

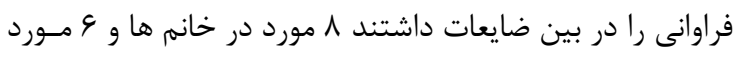

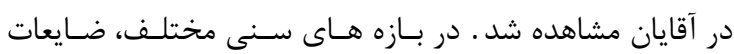

جدول ا: فراوانى و درصد ضايعات غدد بزاقى بر حسب جنسيت، بازه هاى سنى، و محل ضايعات.

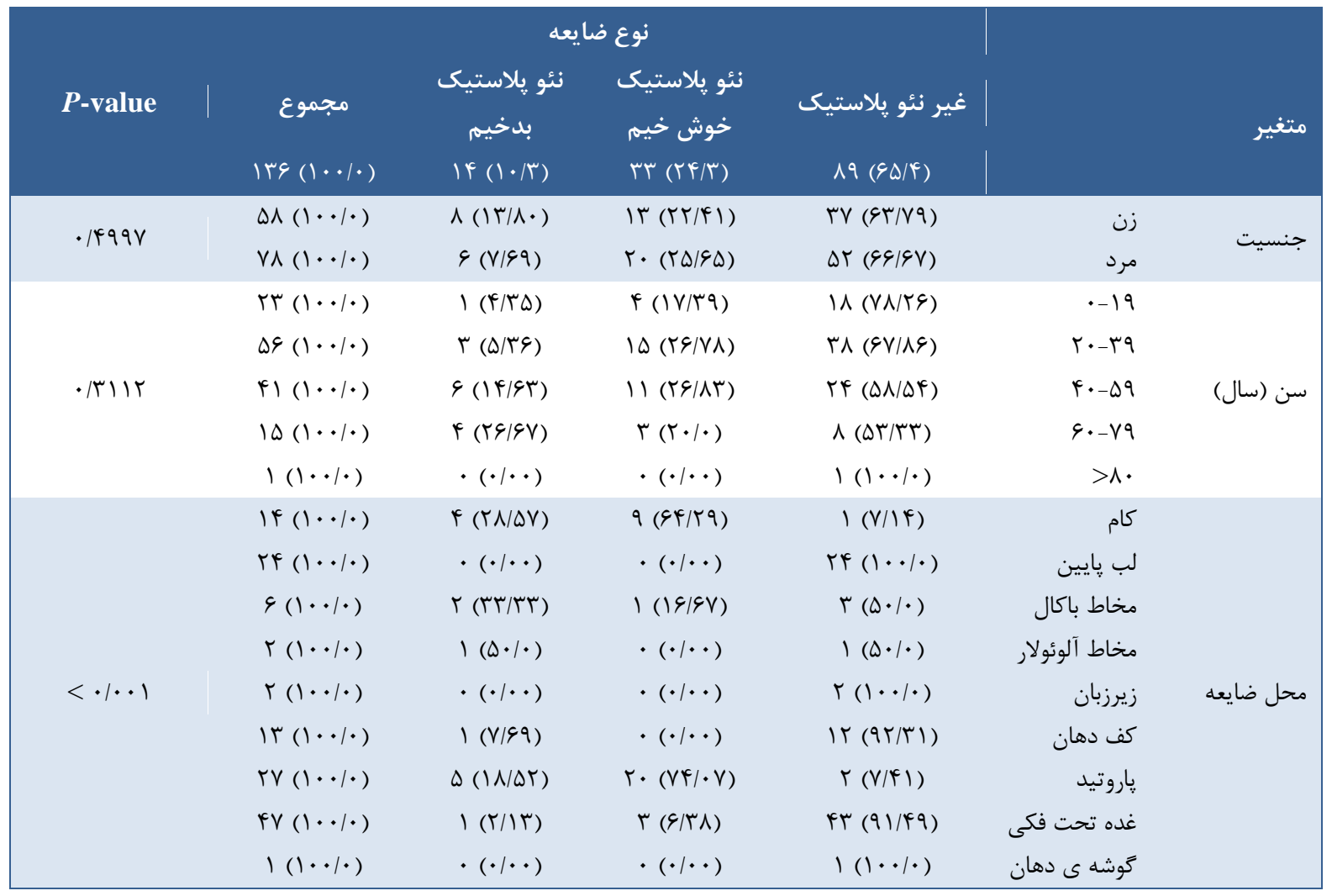


ايجاد شده بود. شايع ترين مكان ها براى ايجـاد يُلئومورفيـى

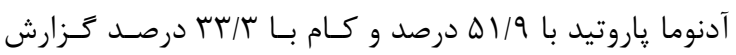

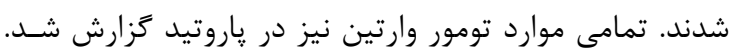

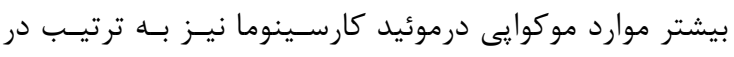

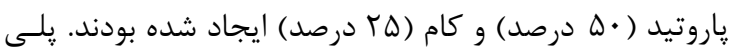

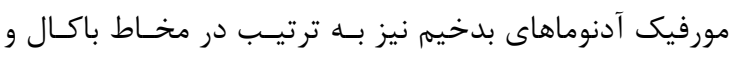

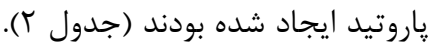

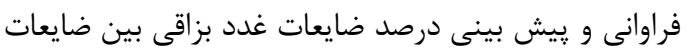

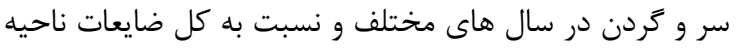

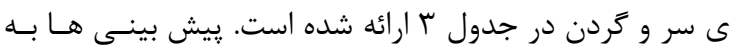
كمك روش هموارسازى نمايى (weighted moving average)

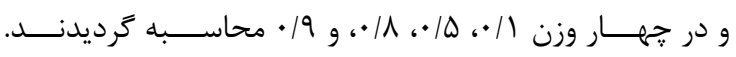

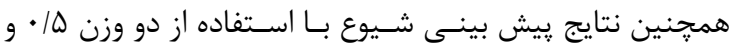

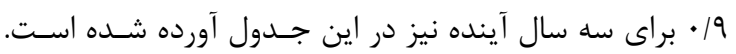

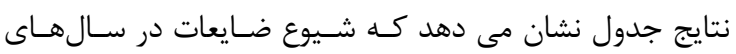

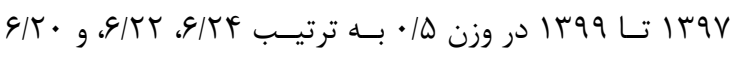

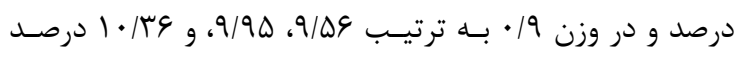

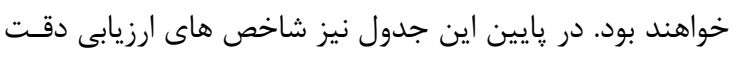

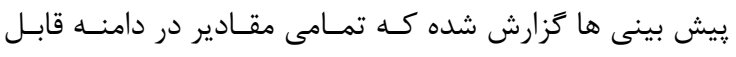

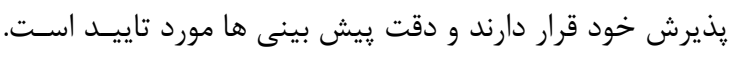

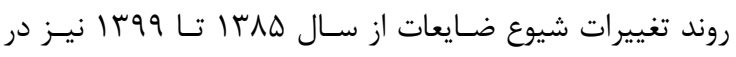
شكل ا نمايش داده شده است.
غيرنئويلاستيك ·F درصد تمامى موكوسل ها در خـانم هـا و

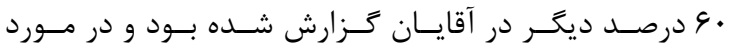

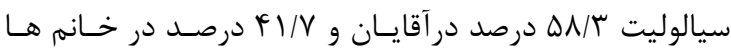

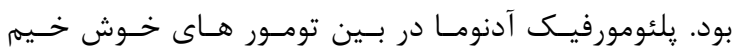

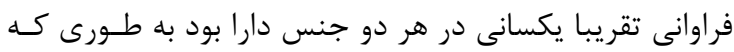

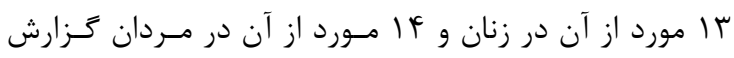

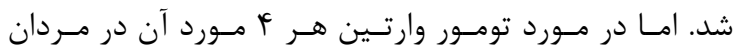

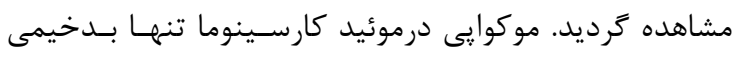

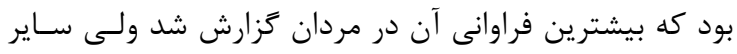

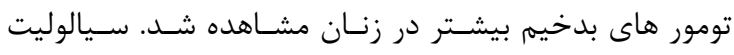

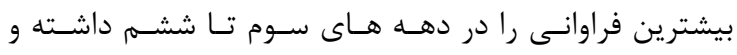

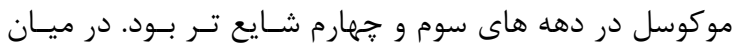

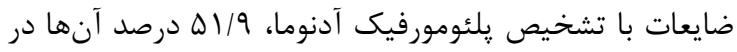

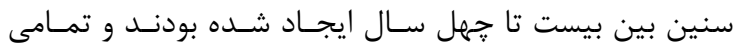

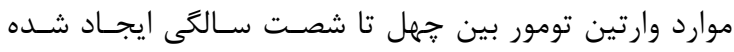

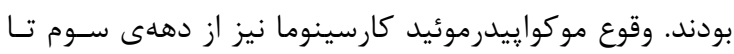

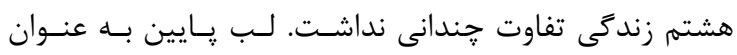

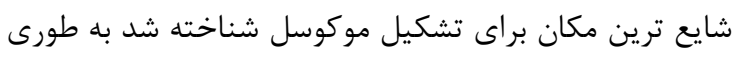

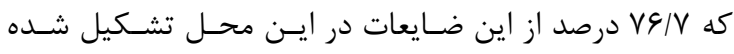

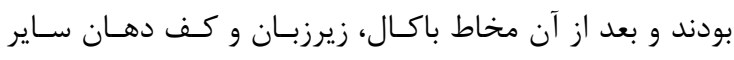

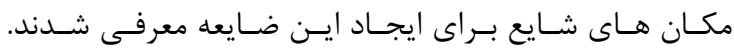

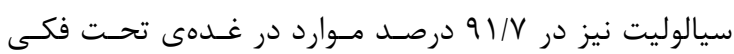

جدول r: فراوانى و درصد انواع ضايعات بر حسب نوع ضايعه، جنسيت و بازه هاى سنى

\begin{tabular}{|c|c|c|c|c|c|c|c|c|c|}
\hline & & سن (سال) & & & سيت & جنس & |فراوانى نوع & & \\
\hline$>\wedge$ & q. -Vq & $p \cdot-\Delta q$ & $r \cdot-r q$ & $\cdot-19$ & مرد & زن & ضايعه & & \\
\hline$\cdot(\cdot / \cdot \cdot)$ & $\cdot(\cdot / \cdot \cdot)$ & $\cdot(\cdot / \cdot \cdot)$ & $1(Y / 9)$ & $1(\Delta / \varphi)$ & $1(1 / 9)$ & $1(Y / 9)$ & $r(T / T)$ & ريتنشن سيست & \\
\hline$\cdot(\cdot / \cdot \cdot)$ & $\cdot(\cdot / \cdot)$ & $1(F / T)$ & $r(\Delta / \Gamma)$ & $1(\Delta / \varphi)$ & $f(V / V)$ & $\cdot(\cdot / \cdot)$ & $f(F / \Delta)$ & سيست مجراى بزاقى & \\
\hline$\cdot(\cdot / \cdot \cdot)$ & $\cdot(\cdot / \cdot \cdot)$ & $V(r q / r)$ & $I V(F F / V)$ & $9(\Gamma / \Gamma)$ & $1 \wedge(T F / \varphi)$ & $\mid r(\Psi / / \varphi)$ & $r \cdot(r r / V)$ & موكوسل & \\
\hline $1(1 \cdot \cdot / \cdot)$ & $\vee(\Lambda \vee / \Delta)$ & $\operatorname{Ir}(\Delta \cdot / \cdot)$ & $11(T / 9)$ & $\Delta(Y V / \Lambda)$ & $|r|(F \cdot / f)$ & $10(\Gamma 9 / \Delta)$ & $r \varphi(f \cdot / \varphi)$ & سيالوليت & غير \\
\hline$\cdot(\cdot / \cdot \cdot)$ & $1(I K / Q)$ & $r(\Lambda / r)$ & $f(1 \cdot \mid \Delta)$ & $\cdot(\cdot / \cdot \cdot)$ & $r(\Delta / \Lambda)$ & $f(1 \cdot \mid \Delta)$ & $V(V / 9)$ & سيالآدنيت & نئويلاستيك \\
\hline$\cdot(\cdot / \cdot \cdot)$ & $\cdot(\cdot / \cdot \cdot)$ & $r(\Lambda / r)$ & $r(V / q)$ & $F(Y T / Y)$ & $\Delta(9 / 9)$ & $\Delta(I r / T)$ & $9(1 \cdot / 1)$ & رانولا & \\
\hline$\cdot(\cdot / \cdot \cdot)$ & $\cdot(\cdot / \cdot \cdot)$ & $\cdot(\cdot / \cdot \cdot)$ & $\cdot(\cdot / \cdot \cdot)$ & $1(\Delta / 9)$ & $(\cdot / \cdot \cdot)$ & $1(Y / 9)$ & $1(1 / 1)$ & سيست نامشخص & \\
\hline $1(1 \cdot \cdot / \cdot)$ & $\wedge(1 \cdot \cdot / \cdot)$ & $\operatorname{rF}(1 \cdots / \cdot)$ & ऍ $(1 \cdots / \cdot)$ & $11(1 \cdot \cdot / \cdot)$ & $\Delta r(1 \cdot \cdot / \cdot)$ & ऍ $(1 \cdot \cdots / \cdot)$ & $\wedge 9(1 \cdots / \cdot)$ & مجموع & \\
\hline$\cdot(\cdot / \cdot \cdot)$ & $r(1 \cdot \cdot / \cdot)$ & $G(\Delta F / \Delta)$ & $\mid f(9 \Psi / \Gamma)$ & $f(1 \cdot \cdot / \cdot)$ & $\operatorname{lf}(V \cdot / \cdot)$ & $\mid r(1 \cdot \cdot / \cdot)$ & $r V(\Lambda) / A)$ & يلئومورفيك آدنوما & \\
\hline$\cdot(\cdot / \cdot \cdot)$ & $\cdot(\cdot / \cdot \cdot)$ & $f(\Psi \varepsilon / F)$ & $\cdot(\cdot / \cdot \cdot)$ & $\cdot(\cdot / \cdot \cdot)$ & $F(r \cdot / \cdot)$ & $\cdot(\cdot / \cdot \cdot)$ & $F(\mid T / I)$ & تومور وارتين & \\
\hline$\cdot(\cdot / \cdot \cdot)$ & $\cdot(\cdot / \cdot \cdot)$ & $\cdot(\cdot / \cdot \cdot)$ & $I(g / V)$ & $\cdot(\cdot / \cdot \cdot)$ & $1(\Delta / \cdot)$ & $\cdot(\cdot / \cdot \cdot)$ & $1(r / \cdot)$ & بازال سل آدنوما & \\
\hline$\cdot(\cdot / \cdot \cdot)$ & $\cdot(\cdot / \cdot \cdot)$ & $1(9 / 1)$ & $\cdot(\cdot / \cdot \cdot)$ & $\cdot(\cdot / \cdot \cdot)$ & $1(\Delta / \cdot)$ & $\cdot(\cdot / \cdot \cdot)$ & $1(\mu / \cdot)$ & كَلندولار تومور* & \\
\hline$\cdot(\cdot / \cdot \cdot)$ & $r(1 \cdot \cdot / \cdot)$ & $11(1 \cdot \cdots / \cdot)$ & $10(1 \cdot \cdots / \cdot)$ & $f(1 \cdot \cdot / \cdot)$ & $r \cdot(1 \cdot \cdot / \cdot)$ & Ir & 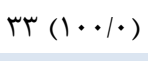 & مجموع & \\
\hline$\cdot(\cdot / \cdot \cdot)$ & $r(\Delta \cdot / \cdot)$ & $r(\Delta \cdot / \cdot)$ & $r(\varnothing 9 / V)$ & $1(1 \cdot \cdot / \cdot)$ & $\Delta(\Lambda \mu / \Gamma)$ & $r(r V / Q)$ & $\wedge(\Delta V / I)$ & موكوايى درموئيد كارسينوما & \\
\hline$\cdot(\cdot / \cdot \cdot)$ & $1(T \Delta / \cdot)$ & $r(T / T)$ & $\cdot(\cdot / \cdot \cdot)$ & $\cdot(\cdot / \cdot \cdot)$ & $1(19 / V)$ & $r(r \Delta / \cdot)$ & $r(T / / F)$ & يلئومورفيك آدنوماى بدخيم & \\
\hline$\cdot(\cdot / \cdot \cdot)$ & $\cdot(\cdot / \cdot \cdot)$ & $\cdot(\cdot / \cdot)$ & | & $\cdot(\cdot / \cdot \cdot)$ & $\cdot(\cdot / \cdot)$ & $1(\mid r / Q)$ & $1(V / I)$ & 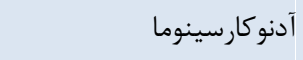 & |نئويلاستيك \\
\hline$\cdot(\cdot / \cdot \cdot)$ & $1(T \Delta / \cdot)$ & $\cdot(\cdot / \cdot \cdot)$ & $\cdot(\cdot / \cdot \cdot)$ & $\cdot(\cdot / \cdot \cdot)$ & $\cdot(\cdot / \cdot \cdot)$ & $1(\mid K / Q)$ & $1(V / 1)$ & كارسينوم مجراى بزاقى & 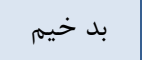 \\
\hline$\cdot(\cdot / \cdot \cdot)$ & $\cdot(\cdot / \cdot \cdot)$ & $1(\mid 9 / V)$ & $\cdot(\cdot / \cdot \cdot)$ & $\cdot(\cdot / \cdot)$ & $\cdot(\cdot / \cdot \cdot)$ & $1(I T / Q)$ & $\mid(V / I)$ & آدنوئيد سيستيك كارسينوما & \\
\hline$\cdot(\cdot / \cdot \cdot)$ & $f(1 \cdot \cdot / \cdot)$ & $9(1 \cdot \cdot / \cdot)$ & $r(1 \cdot \cdot / \cdot)$ & $1(1 \cdot \cdot / \cdot)$ & $9(1 \cdots / \cdot)$ & $\wedge(1 \cdot \cdot / \cdot)$ & If $(1 \cdots / \cdot)$ & مجموع & \\
\hline
\end{tabular}


جدول س: فراوانى و پيشى بينى ضايعات غدد بزاقى بين ضايعات سر و كردن در سال هاى مختلف و نسبت به كل ضايعات ناحيه سى سر و كردن

\begin{tabular}{|c|c|c|c|c|c|c|c|}
\hline $\begin{array}{c}\text { Trial } 4 \\
\cdot / 9\end{array}$ & $\begin{array}{c}\text { Trial } 3 \\
\cdot / \wedge\end{array}$ & $\begin{array}{c}\text { Trial } 2 \\
\cdot / \Delta\end{array}$ & $\begin{array}{c}\text { Trial } 1 \\
. / 1\end{array}$ & شيوع & تعداد ضايعات غدد & هاى برراد كل برونده شده & سال \\
\hline$G / V \Delta$ & $G / V \Delta$ & $G / V \Delta$ & $G / V \Delta$ & $T / \Delta F$ & $r$ & 111 & $\| \wedge \Delta$ \\
\hline र/१९ & $\Gamma / \Gamma \wedge$ & $F / 90$ & r/א & q/Ar & 11 & 114 & IrAs \\
\hline $9 / 14$ & N/Dr & $\Delta / F q$ & $8 \mid 9 \wedge$ & $1 \% / 99$ & TI & 194 & IrAV \\
\hline $\mid r / \Delta \Lambda$ & $\mid r / \cdot V$ & $4 \mid \cdot \Lambda$ & $V / T)$ & $p / r q$ & $\wedge$ & INT & $\| r \wedge \Lambda$ \\
\hline$\Delta / r)$ & ه/१ & $9 / 99$ & $V / \cdot 1$ & $F / \wedge \Delta$ & 1. & $r \cdot G$ & $1 \% \wedge 9$ \\
\hline r/A & $\Delta / \cdot G$ & ધ|^૬ & $\varphi / \Lambda$. & $r / r \wedge$ & $\wedge$ & $1 \wedge V$ & $1 \% q$. \\
\hline F/MF & $F / F F$ & G/Ar & $\varepsilon / \Delta \Delta$ & r/lq & 9 & 19. & $\mid r q 1$ \\
\hline$\Gamma / T \Lambda$ & $r / f \mid$ & $9 / 99$ & $s|r|$ & $r /|q|$ & V & $r \cdot \Delta$ & Irar \\
\hline$r / F$. & $r / F \mid$ & $\varepsilon / F \Delta$ & ه/q & N/99 & $r$. & ו & וrq \\
\hline$N / I r$ & $V|\varphi|$ & $9 / 19$ & $q / T$. & q/बए & TI & TIN & $1 r q 4$ \\
\hline $9 / 4 \wedge$ & $9 / r \mu$ & $9 / 19$ & $\varepsilon \mid \Delta F$ & $\Lambda / \Lambda$. & IV & 194 & $1 \pi q \Delta$ \\
\hline N/AV & ^/^ৎ & $\varepsilon / T V$ & $9 / \wedge$. & $N / D \mid$ & f & iv & 1 I १९ \\
\hline & & & & G|GT & $1 \pi 4$ & $r \cdot \Delta I$ & مجموع \\
\hline & & & & \multicolumn{4}{|c|}{ ييش بينى ها براى سه سال آينده } \\
\hline $9 / \Delta \varphi$ & & G/TF & & $N / \Delta 9$ & & & IrqV \\
\hline $9 / 9 \Delta$ & & G/TT & & $N / F V$ & & & 1491 \\
\hline $1 \cdot / \pi^{4}$ & & $q / T$. & & N/rG & & & 11499 \\
\hline \multicolumn{8}{|c|}{ شاخص هاى ارزيابى دقت يِيش بينى ها } \\
\hline$p / q$. & & $1 / V T$ & & r/बV & متوسط خطاى نسبى & & \\
\hline$\cdot / 1 \mathrm{~V}$ & & $\cdot / \Delta \mu$ & & .119 & نسبت واريانس (C) & & \\
\hline$\cdot / 90$ & & .191 & & .199 & احتمال خطا (P) & & \\
\hline
\end{tabular}

\section{$\longrightarrow$ Prevalence}

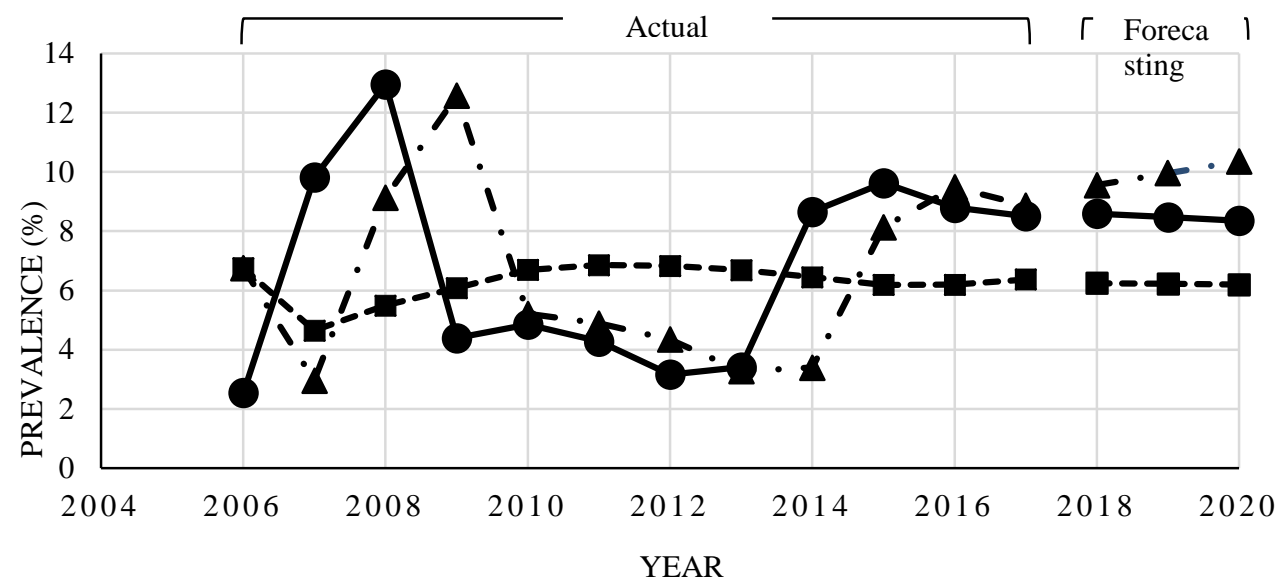

شكل ا: نمودار شيوع هاى مشاهده شده و ييش بينى شيوع ضايعات سه سال آينده با استفاده از روش سرى زمانى هموارسازى نمايى

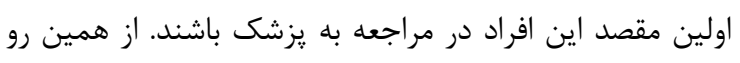

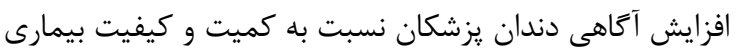

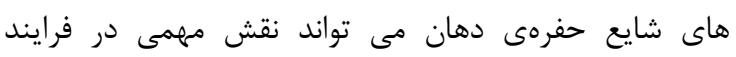

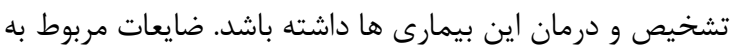

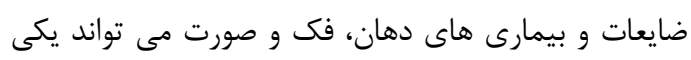

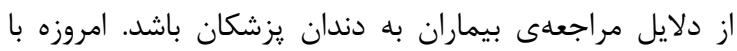

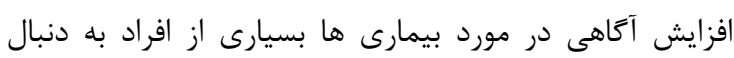

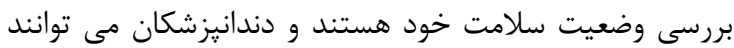


درصد كزارش شد [Yr]. كه با نتايج مطالعهى ما در اين مورد تقريبا همسو مى باشد در مطالعهى Mohajerani و هم همكاران

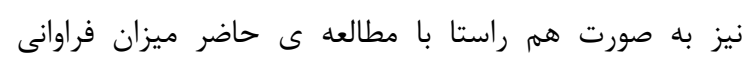

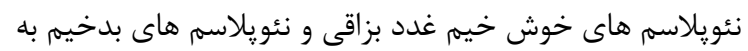

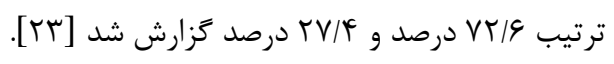

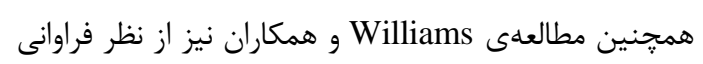

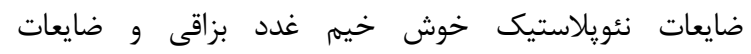

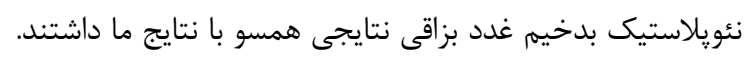

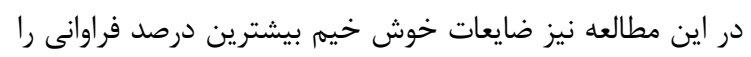

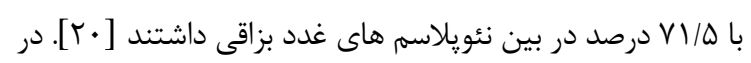

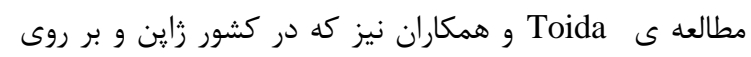

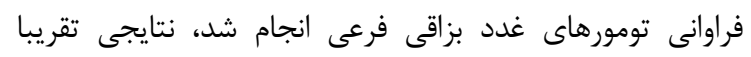

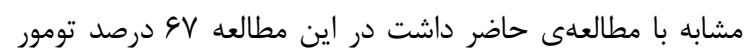

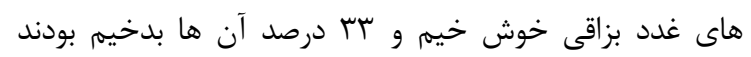

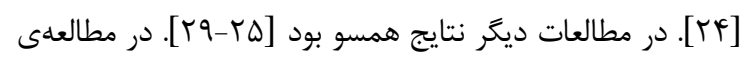

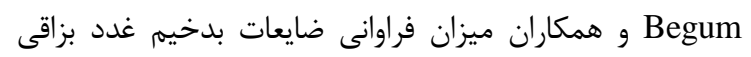

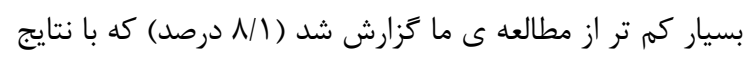

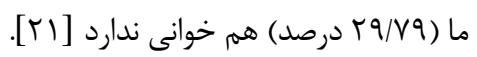
از لحاظ بررسى ضايعات مورد مطالعه در رابطه با جنائ جنسيت،

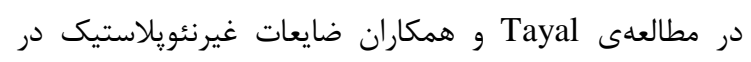

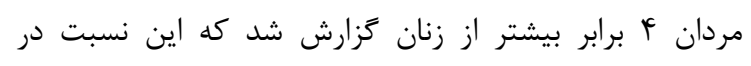

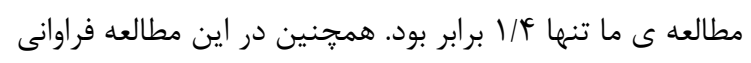

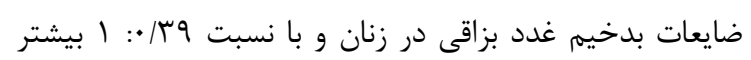

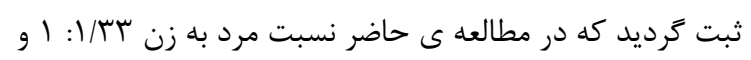

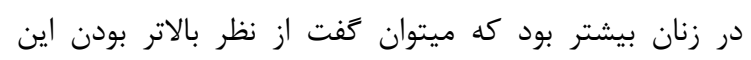

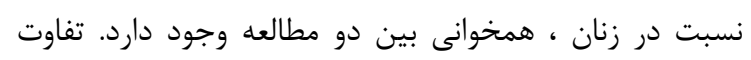

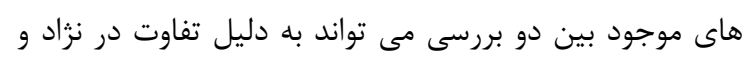

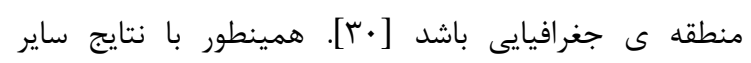

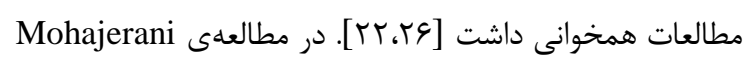

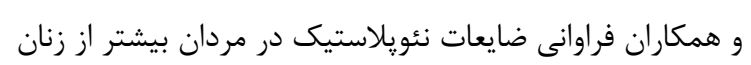

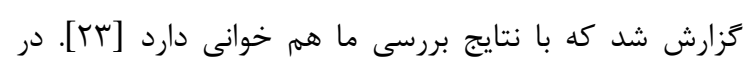

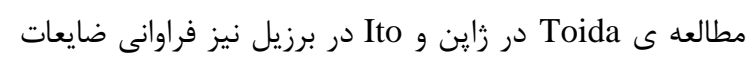

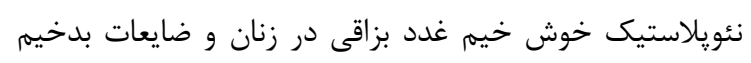

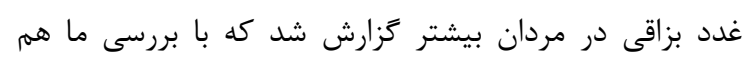

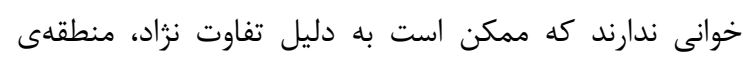

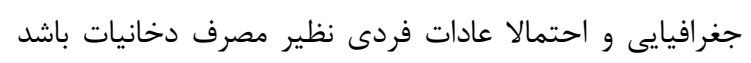

[TY.TO]

از نظر بررسى فراوانى ضايعات غدد بزاقى بر حسب سب سن،

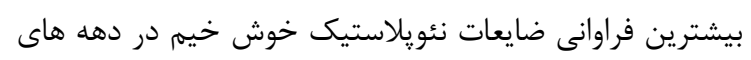

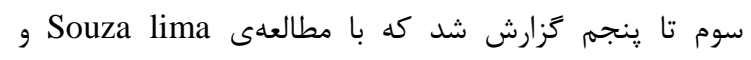

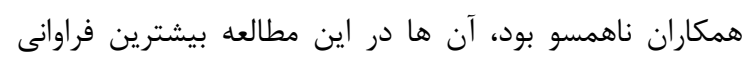

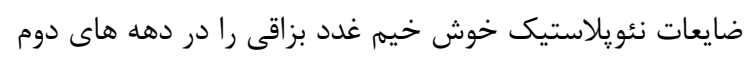

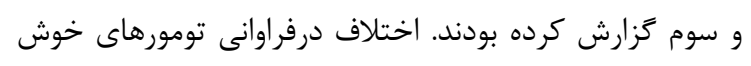

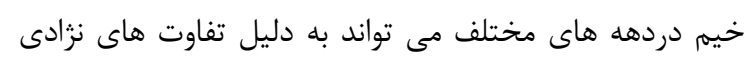

غدد بزاقى از اين دسته بيمارى ها مى باشند كه هركونه تغيير

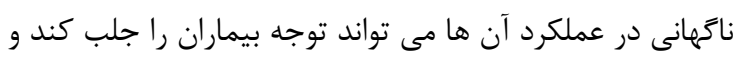

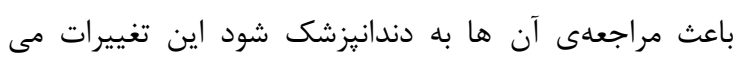

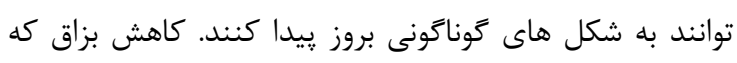

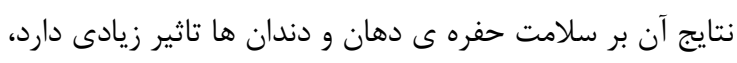

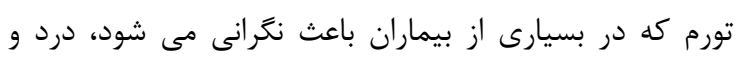

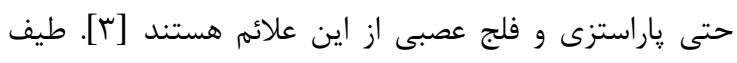

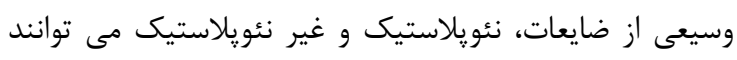

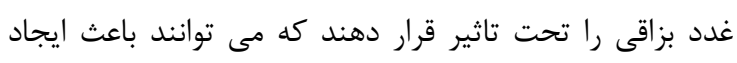

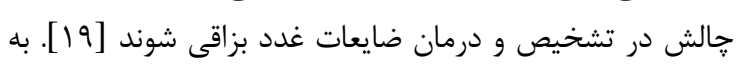

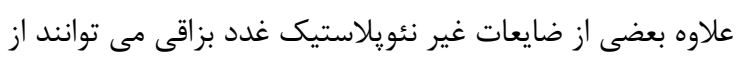

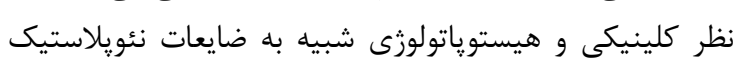

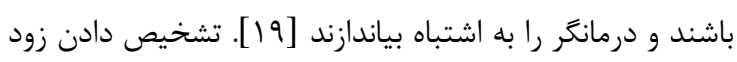

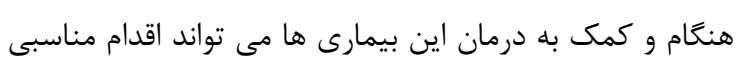

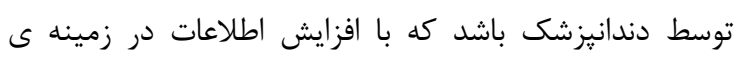
إيدميولوزى بيمارى ها اين امر حاصل مى دئ شود.

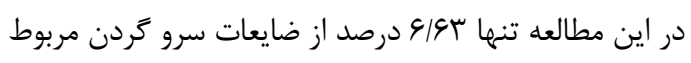

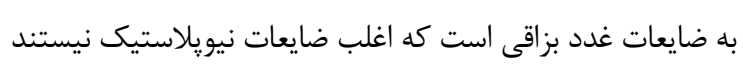

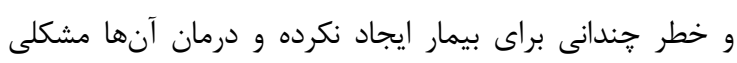

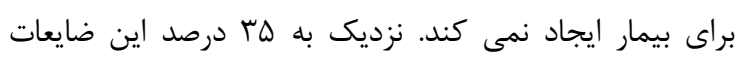

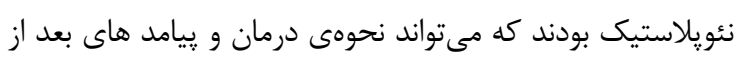

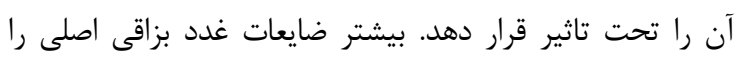

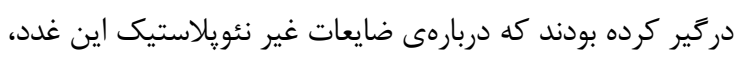

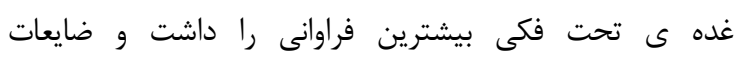

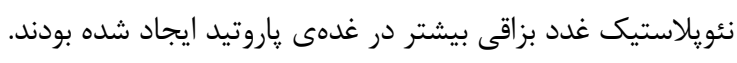

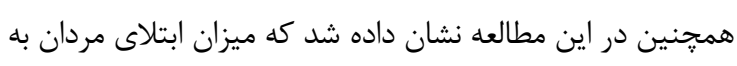

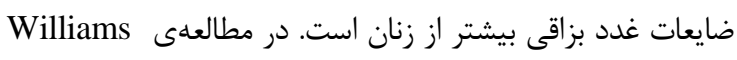

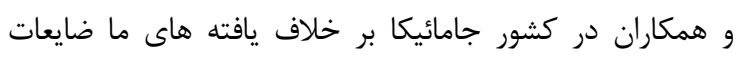

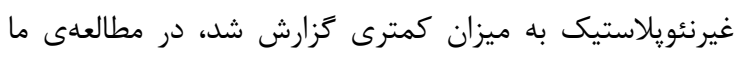

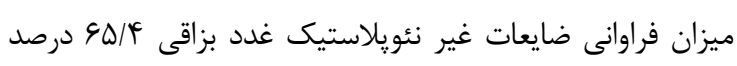

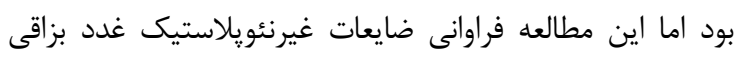

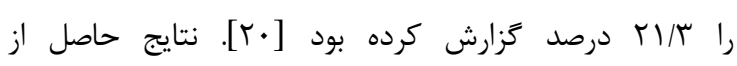
مطالعلى Begum و همكاران در بنحكلادش كه تنها

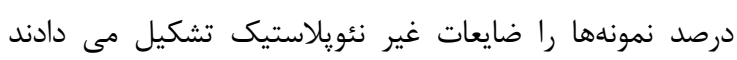

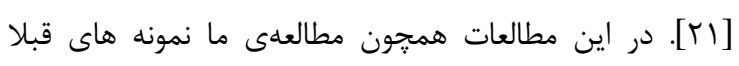

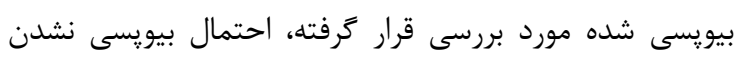

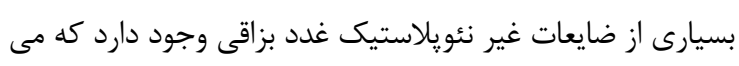

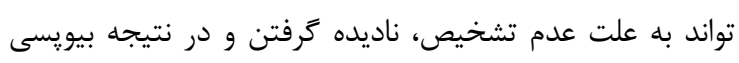

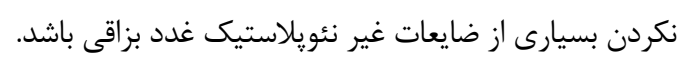

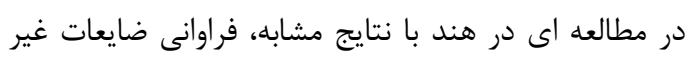

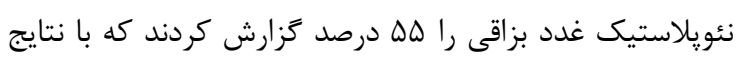

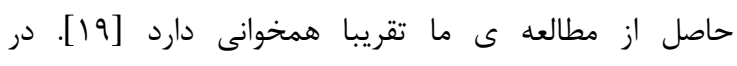

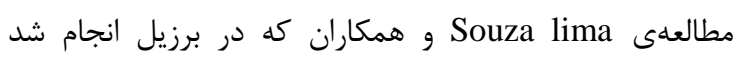

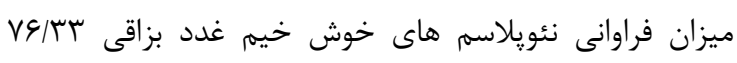




$$
\begin{aligned}
& \text { اطلاعات كافى از جمله محل زندگى، شغل، وضعيت اجتماعى و }
\end{aligned}
$$

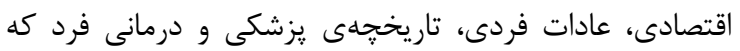

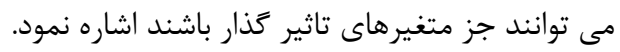

نتيجه كيرى

در اين مطالعه مشخص شد كه از كل مراجعين طى سال

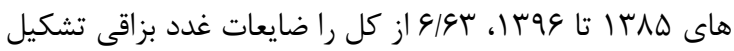
مى دهند. كه بيشترين فراوانى در ضايعات غيرنئويلاستيك ردات

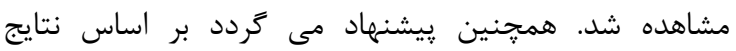

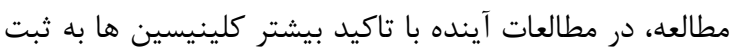

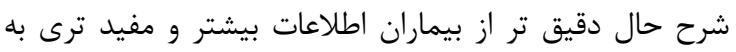

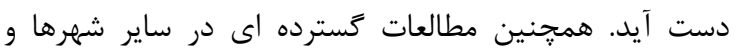
استان ها و بازه إى زمانى طولانى ترى انجام گيرد.

$$
\begin{aligned}
& \text { تشكر و قرو فانى } \\
& \text { نويسندكان اين مقاله بر خود لازم مى دارى دانند، از يشتيبانى }
\end{aligned}
$$

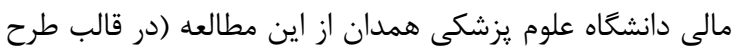

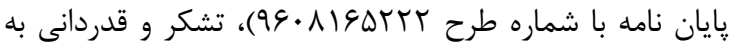

$$
\begin{aligned}
& \text { عمل آورند. - ماند }
\end{aligned}
$$

تضاد منافع

نويسند

\section{REFERENCES}

1. Khoshnevisan MH. Oral health \& community dentistry . $1^{\text {st }}$ ed . Iran : Jahad daneshgahi publications ; 2016.p.82-83.

2. Madhu B.S, Vipin Das P.R . Clinico-Pathological Study \& Management of Parotid Gland Tumors. Journal of Dental and Medical Science . 2015;4(4): 48-54 .

3. Epker BN. Obstructive and inflammatory diseases of the major salivary glands. Oral Surgery, Oral Medicine, Oral Pathology. 1972;33(1):2-7. DOI.org/10.1016/0030-4220(72) 90203-4

4. Stenner M, Klussmann JP. Current update on established and novel biomarkers in salivary gland carcinoma pathology and the molecular pathways involved. European Archives of Oto-Rhino-Laryngology. 2009;266(3):333-41. DOI.org/10. 1007/s00405-008-0882-7

5. Rogers J, McCaffrey TV. Inflammatory disorders of the salivary glands. Otolaryngology: Head \& Neck Surgery. 5th ed. Philadelphia: Mosby Elsevier; 2010. p.1333-43.

6. Berndt AL, Buck R, von Buxton RL. The Pathogenesis Of Acute Suppurative Parotitis. The American journal of the Medical Sciences. 1931;182(5):639-49.

7. Wilson KF, Meier JD, Ward PD. Salivary gland disorders. American family physician. 2014;89(11): 882-888.

8. Arduino PG, Carrozzo M, Pentenero M, Bertolusso G, Gandolfo S. Non-neoplastic salivary gland diseases. Minerva stomatologica. 2006;55(5):249-70.

9. Epker BN. Obstructive and inflammatory diseases of the major salivary glands. Oral Surgery, Oral Medicine, Oral Pathology. 1972;33(1):2-7. DOI.org/10.1016/0030-4220(72) 90203-4

10. Pederson AM, Reibel J. Primary Sjogren's syndrome (pSS): subjective symptoms and salivary findings. Journal of Oral Pathology and Medicine. 1999; 28: 303-311. DOI.org/10.1111/j.1600-0714.1999.tb02045.x

11. Balachander N, Jimson S, Mastha K . Non-neoplastic Salivary Gland Diseases . Biomedical \& Pharmacology Journal . 2013; 6(2): 385-388 . DOI.org/10.13005/bpj/431
در جامعه ى مورد مطالعه و همجنين تغيير عادات فردى افراد

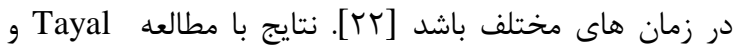

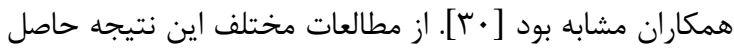

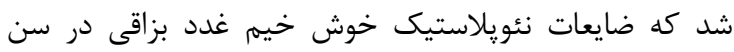

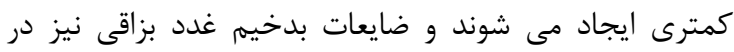

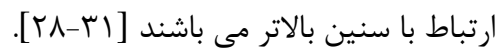

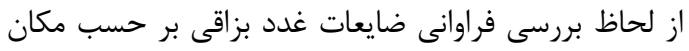

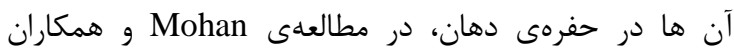

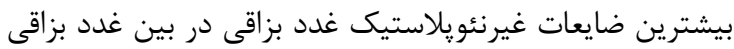

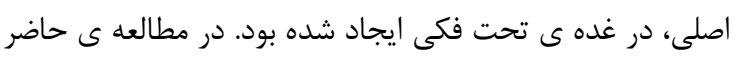

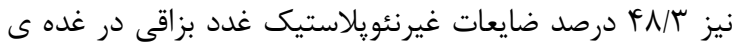
تحت فكى ايجاد شده بودند، ميزان بالاى اين فراوانى به علت

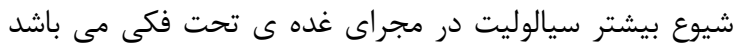

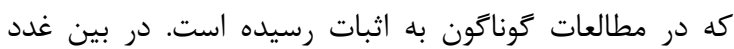

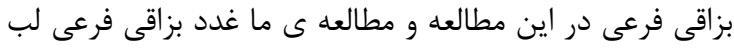

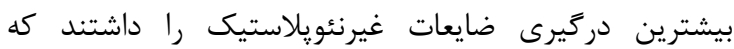

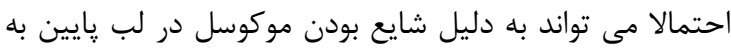

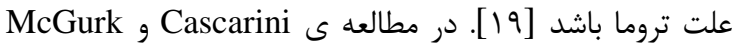

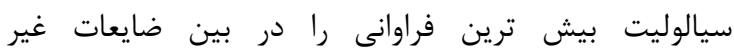

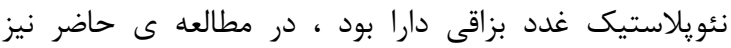

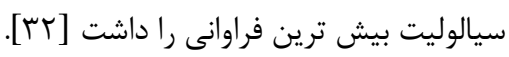
از محدوديت هاى اين مطالعه مى توان به عدم ثبت بت ريت

12. McQuone SJ. Acute viral and bacterial infections of the salivary glands. Otolaryngologic Clinics of North America. 1999;32(5):793-811. DOI.org/10.1016/S00306665(05)70173-0

13. Neville BW, Damm DD, Chi AC, Allen CM. Oral and maxillofacial pathology -E-Book. 4th Ed . St.Louis: Elsevier ; 2016.p.422-72.

14. Kayembe MK, Kalengayi MM. Salivary gland tumours in Congo (Zaire). Odonto-stomatologie tropicale, Tropical dental journal. 2002;25(99):19-22

15. Shishegar M, Ashraf MJ, Azarpira N, Khademi B, Hashemi B, Ashrafi A. Salivary gland tumors in maxillofacial region: a retrospective study of 130 cases in a southern Iranian population. Pathology research international. 2011;2011; 15. DOI.org/10.4061/2011/934350

16. Licitra L, Grandi C, Prott FJ, Schornagel JH, Bruzzi P, Molinari R. Major and minor salivary glands tumours. Critical reviews in oncology/hematology. 2003;45(2):21525.DOI.org/10.1016/S1040-8428(02)00005-7

17. Pour H, Zarei MR, Chamani G, Rad M. Malignant salivary glands tumors in Kerman province: a retrospective study. Dental Research Journal. 2008;4(1): 4-10.

18. Wang D, Li Y, He H, Liu L, Wu L, He Z. Intraoral minor salivary gland tumors in a Chinese population: a retrospective study on 737 cases. Oral Surgery, Oral Medicine, Oral Pathology, Oral Radiology, and Endodontology. 2007;104(1):94-100. DOI.org/10.1016/j. tripleo.2006.07.012

19. Mohan H, Tahlan A, Mundi I, Punia RP, Dass A. Nonneoplastic salivary gland lesions: a 15-year study. European Archives of Oto-Rhino-Laryngology. 2011;268(8):1187-90. DOI.org/10.1007/s00405-010-1460-3

20. Williams NP, Boyd DL, Choy L, Hanchard B. Salivary gland lesions: a Jamaican perspective. West Indian Med J. 2001;50(1):62-5.

21. Begum A, Baten MA, Alam MM, Huq MH, Ahsan 
MM, Khan MK, Saleh FM, Talukder S. Spectrum of Salivary Gland Lesions in a Tertiary Level Hospital. Mymensingh Med J. 2015 ;24(3):516-20.

22. Lima SS, Soares AF, Amorim RF, Almeida FR. Epidemiologic profile of salivary gland neoplasms: analysis of 245 cases. Brazilian journal of otorhinolaryngology. 2005;71(3):335-40. DOI.org/10.1016/S1808-8694(15)31332-X

23. Mohajerani H, Ghatan MT, Khojasteh A, Mohajerani SA. A ten years epidemiologic study of salivary gland tumors in Loghman Hospital. journal of Dental School. 2009; 27(3):155-59

24. Toida M, Shimokawa K, Makita H, Kato K, Kobayashi A, Kusunoki Y, atakeyama D, Fujitsuka H, Yamashita T, Shibata T. Intraoral minor salivary gland tumors: a clinicopathological study of 82 cases. International journal of oral and axillofacial surgery. 2005;34(5):528-32. DOI.org/10.1016/j.ijom.2004.10.010

25. Ito FA, Ito K, Vargas PA, De Almeida OP, Lopes MA. Salivary gland tumors in a Brazilian population: a retrospective study of 496 cases. International journal of oral and maxillofacial surgery. 2005;34(5):533-6. DOI.org/10.1016/j.ijom.2005.02.005

26. Bobati SS, Patil BV, Dombale VD. Histopathological study of salivary gland tumors. Journal of oral and maxillofacial pathology: JOMFP. 2017;21(1):46. DOI: 10.4103/0973029X.203762

27. Buchner A, Merrell PW, Carpenter WM. Relative frequency of intraoral minor salivary gland tumors: a study of 380 cases from northern California and omparison to reports from other parts of the world. Journal of oral pathology \& medicine. 2007;36(4):207-14. DOI.org/10.1111/j.16000714.2007.00522. $\mathrm{x}$

28. Adebiyi KE, Emmanuel MM. Neoplastic Salivary Gland Lesions: A Retrospective Analysis of 135 Cases from Lagos State University Teaching Hospital, Ikeja, Lagos, Nigeria. West African journal of medicine. 2014;33(3):206-10.

29. Xu B, Aneja A, Ghossein R, Katabi N. Salivary gland epithelial neoplasms in pediatric population: a singleinstitute experience with a focus on the histologic spectrum and clinical outcome. Human Pathology. 2017;67:37-44. DOI.org/10.1016/j.humpath.2017.07.007

30. Tayal U, Bajpai M , Jain A, Dhupia J . Fnac of salivary gland lesion-study of 126 cases at a tertiary care center of national capital region india. Journal of Medical and Dental Science Research . 2014;1(2): 04-06

31. Buchner A, Merrell PW, Carpenter WM. Relative frequency of intraoral minor salivary gland tumors: a study of 380 cases from northern California and omparison to reports from other parts of the world. Journal of oral pathology \& medicine. 2007;36(4):207-14. DOI.org/10.1111/j.16000714.2007.00522.x

32. Cascarini L, McGurk M. Epidemiology of salivary gland infections. Oral and maxillofacial surgery clinics of North America. 2009;21(3):353-7. DOI.org/10.1016/j.coms. 2009.05.004 